\title{
Dos nuevos Registros de Habenaria (Orchidaceae) PARA LA FLORA ARGENTINA
}

\author{
CHRISTIAN A. ZANOTTI'1 , JOSÉ A. RADINS² y HÉCTOR A. KELLER 3
}

\begin{abstract}
Summary: Two new records of Habenaria (Orchidaceae) for the Argentinian flora. Habenaria johannensis and $H$. josephensis, species known up to now with the distribution limit in southern Brazil, are cited for the first time for northeastern Argentina. A brief description of the species, geographical distribution, ecological observations, field photographs, a map with the distribution of the two new records in Argentina, as well as a key to differentiate the species present in northeastern of Argentina are provided.
\end{abstract}

Key words: Corrientes province; Misiones province, northeastern Argentina, terrestrial orchids.

\begin{abstract}
Resumen: Se cita por primera vez para el nordeste de la Argentina a Habenaria johannensis y $H$. josephensis, especies conocidas hasta el momento con el límite de distribución más austral en el sur del Brasil. Se aportan una breve descripción de las especies, distribución geográfica, observaciones ecológicas, fotografías de campo, un mapa con la distribución de los dos nuevos registros en la Argentina, así como también, una clave para diferenciar las especies de Habenaria presentes en el nordeste argentino.
\end{abstract}

Palabras clave: Noreste de Argentina, orquídeas terrestres, provincia de Corrientes, provincia de Misiones.

\section{INTRODUCCIÓN}

Habenaria Willd., es uno de los géneros más importantes en cuanto al número de especies dentro de la familia Orchidaceae compuesto por alrededor de 881 especies (Govaerts et al., 2018) de distribución cosmopolita, que habita en regiones tropicales y subtropicales, con algunas especies alcanzando regiones templadas, particularmente en el Asia oriental (Pridgeon et al., 2001); es probablemente de origen africano (Batista et al., 2013) y con centros de diversificación primarios en Brasil, sur y centro de África y este de Asia (Kurzweil \& Weber, 1992).

1 Instituto de Botánica Darwinion, IBODA-CONICET, Casilla de Correo 22, B1642HYD, San Isidro, Buenos Aires, Argentina. E-mail: czanotti@darwin.edu.ar.

${ }^{2}$ Ministerio de Ecología y Recursos Naturales Renovables de Misiones. Vivero de Especies Nativas. Candelaria, Misiones, Argentina.

${ }^{3}$ Instituto de Botánica del Nordeste, UNNE-CONICET, Casilla de Correo 209, 3400, Corrientes, Argentina.
Este género se encuentra representado por plantas geófitas perennes con un ciclo de crecimiento y reproducción vinculada a una temporada húmeda seguida de una seca que es cuando los individuos persisten como tubérculos subterráneos (Batista et al., 2013). Se caracteriza por presentar pétalos generalmente 2-partidos no fusionados a otras partes de la flor, labelo 3-partido que carece de callo, lóbulos del estigma libres, enteros, no adnatos a los pétalos o al labelo, espolón nectarífero desarrollado (Dressler, 1993; Pridgeon et al., 2001) y anteras con una profunda división que posteriormente resulta en la formación de dos polinios separados en la mayoría de las especies (Singer \& Cocucci, 1997).

La integridad de este género ha sido cuestionada por más de un siglo por diferentes autores como así también su monofilia (Bateman et al., 2003) proponiendo la segregación del mismo en diversos géneros y/o en diferentes clasificaciones infragenéricas (ej. Batista et al., 2011a; Pedron et al., 2014). Recientemente, Batista et al. (2013) llevaron a cabo un estudio filogenético molecular de las especies de Habenaria del Nuevo Mundo, 
el cual reveló que los representantes neotropicales forman un grupo monofilético que consta de al menos 21 subgrupos bien sustentados, y que son parafiléticas o polifiléticas todas las secciones neotropicales de la actual clasificación (Kränzlin, 1892, 1901; Cogniaux, 1893). Por esta razón, Batista et al. (2013) propusieron realizar una amplia revisión taxonómica del género y recircunscribir las secciones establecidas previamente, como se ha llevado a cabo actualmente en los representantes de la sección Pentadactylae Kraenzl. (Pedron et al., 2014) y la sección Macroceratitae Kraenzl. (Batista et al., 2006).

El número de especies de Habenaria reportadas para la Argentina varía desde 15 (Batista et al., 2011a, b) hasta 20 (Schinini et al., 2008) sin ningún endemismo nacional. Estudios florísticos recientes realizados en las provincias de Corrientes y Misiones nos han permitido hallar dos especímenes cuyas características se ajustan a la descripción de H. johannensis Barb. Rodr., especie solo conocida para Bolivia, Brasil y Paraguay, y H. josephensis Barb. Rodr., especie conocida como endémica de Brasil (Batista et al., 2011a).

\section{Materiales y Métodos}

Para constatar los nuevos registros, se comparó el material coleccionado con las descripciones originales y con el material tipo disponible en la Swiss Orchid Foundation at the Herbarium Jany Renz (https://orchid.unibas.ch/index.php/en/ database-search/advanced-search?reset=1). Las descripciones de las especies se basaron en el material colectado para el área y se compararon con las realizadas en trabajos taxonómicos previos (Batista et al., 2006; Vieira \& Barros, 2017). En la clave taxonómica se incluyeron las especies citadas en la Argentina y compartidas con el sur del Brasil de la sinopsis realizada por Batista et al. (2011a,b): $H$. achalensis Kraenzl., H. araneiflora Barb. Rodr., $H$. aranifera Lindl., H. bractescens Lindl., $H$. ekmaniana Kraenzl., H. gourlieana Gillies ex Lindl., $H$. guilleminii Rchb. f., $H$. hieronymi Kraenzl., H. parviflora Lindl., $H$. pentadactyla Lindl., H. repens Nutt. y H. trifida Kunth. Estas especies fueron incluidas en la clave, ya que en la mayoría de los casos, en el trabajo mencionado no se especificó la distribución de dichas especies dentro de la Argentina y que pueden presentarse en el área de estudio. Además, se consideraron las especies citadas para el nordeste argentino (Provincias de Chaco, Corrientes, Formosa y Misiones) por Schinini et al. (2008): H. hassleriana Cogn., $H$. petalodes Lindl. y $H$. regnellii Cogn. Se excluyeron las especies $H$. secunda Lindl., $H$. paiveana Rchb. F. y H. rupicola Barb. Rodr., ya que según Batista et al. (2011a,b) corresponderían a materiales mal identificados.

\section{Resultados}

Como resultado del trabajo se confirma la presencia en la Argentina de $H$. johannensis y $H$. josephensis para las cuales se proveen el tratamiento taxonómico de cada una, descripciones morfológicas, un mapa con la distribución de las especies en el país y fotografías de campo tanto de las especies estudiadas como de algunas otras presentes en el área de estudio. Además, se confeccionó por primera vez una clave para la identificación de las especies de Habenaria que habitan en el nordeste argentino.

\section{Tratamiento taxonómico}

1. Habenaria johannensis Barb. Rodr. Revista Engen. 3: 74, est. 2, fig. B. 1881. Tipo. Barbosa Rodrigues, Iconographie des Orchidées $d u$ Brésil 1: tab. 6 B. 1996 (lectotipo designado por Batista et al., Harvard Pap. Bot. 2011a: 38. (Fig. 1A-C).

Hierbas terrestres de 70-150 $\mathrm{cm}$ de altura. Tubérculos elipsoides u ovoides de 3-6 × 2-3 $\mathrm{cm}$. Hojas lineares de 10-35 × 1-3 cm, de ápice agudo, membranáceas. Inflorescencias laxas, de 15-30 cm de long.; brácteas lanceoladas de 3,5-6,5 $\times 1,5(-2) \mathrm{cm}$, de ápice agudo. Flores verdoso-blanquecinas; sépalo dorsal ovadolanceolado a largamente lanceolado, de 12 $22 \times 10-12 \mathrm{~mm}$, apiculados; sépalos laterales oblicuamente oblongo-lanceolados, levemente falcados, de 20-25 × 6-10 mm, apiculados; pétalos 2-partidos, segmento posterior oblongolanceolado, levemente falcado, connivente con el sépalo dorsal, de 16-20 × 4-7 mm, de ápice obtuso, segmento anterior linear de 25-40 × 1-1,5 


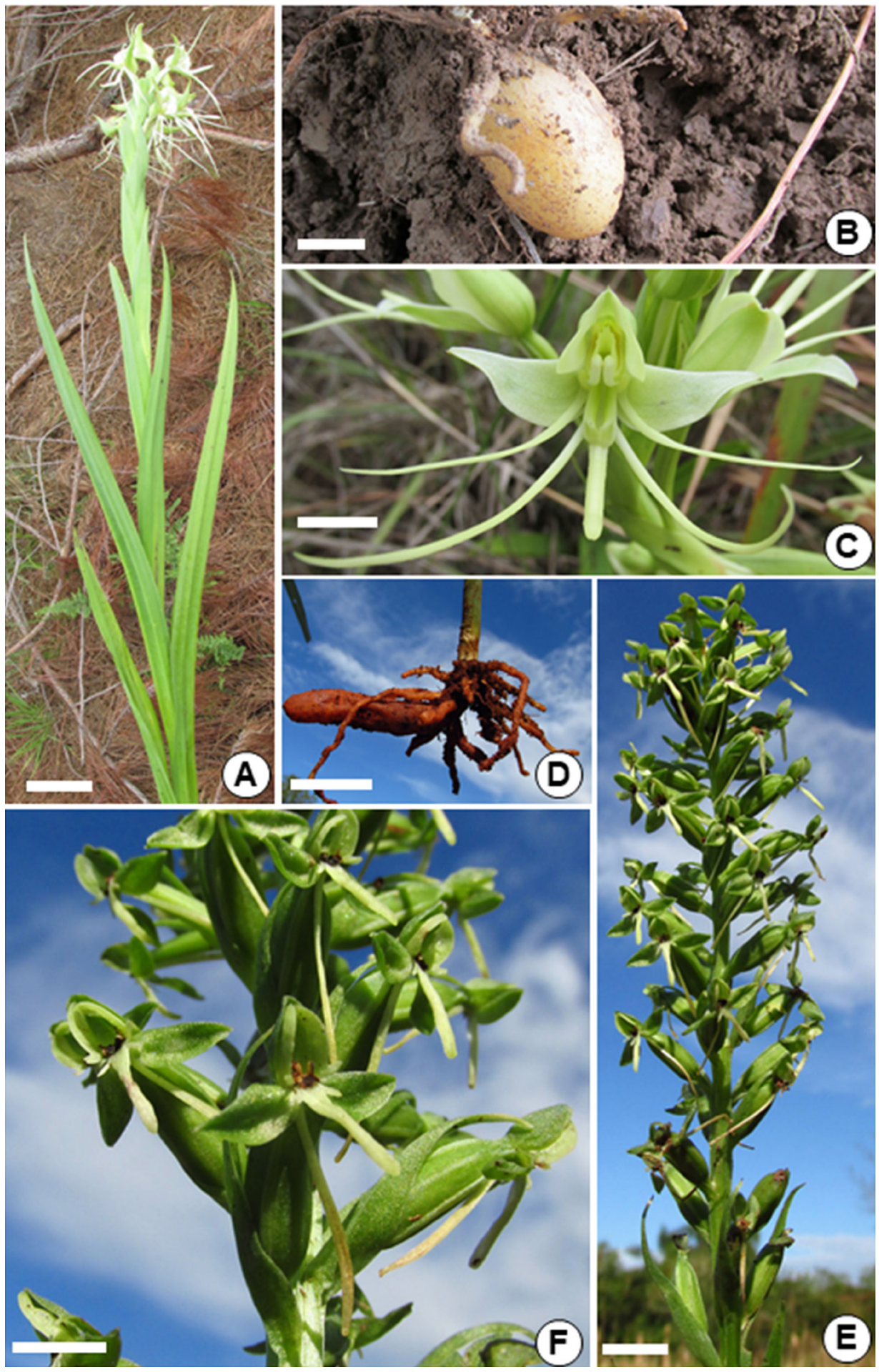

Fig. 1. A-C: Habenaria johannensis. A: Planta. B: Tubérculo. C: Flor. D-F: H. josephensis. D: tubérculo. E: Inflorescencia. F: Flor. Escalas = A: $3 \mathrm{~cm}$; B: $1,5 \mathrm{~cm}$; C: 0,7 cm; D: 2,5 cm; E: 0,6 cm; F: 0,11 cm. Fotos: $\mathrm{H}$. A. Keller. 
mm, curvados, paralelos a los segmentos laterales del labelo, de ápice agudo; labelo 3-partido, segmentos laterales lineares, de 30-50 × 0,8-1 $\mathrm{mm}$, segmento medio linear-ligulado, de 15-25 $\times$ 1,3-2 mm, espolón pendiente, linear, de 11-14 cm de long. Gimnostemio de 7-8 mm de long.; ovario y pedicelo de ca. 1,5 cm de long.; lóbulo medio del rostelo prominente, generalmente proyectado más allá de las anteras, de ápice ancho, dentado. Fruto cápsula 3-costada, de 3-5 × 1,7-2 cm.

Iconografía. Batista et al. (2006: 14, fig. 1C; 19, fig. 4C; 23, fig. 5A).

Distribución geográfica, hábitat $y$ observaciones ecológicas. H. johannensis se encuentra con anterioridad registrada para Bolivia, Brasil y Paraguay (Batista et al., 2006, 2011). Esta especie es típica de ambientes húmedos, creciendo en prados, así como también en ambientes inundados o a lo largo de los márgenes de los ríos y lagos (Batista et al., 2006). Ocasionalmente se la puede encontrar colonizando bancos húmedos al costado de las rutas y otros ambientes modificados (Batista et al., 2006).

En la Argentina (provincia de Misiones; Fig. 2) ha sido hallada en pastizales de suelos húmedos, asociados a afloramientos de areniscas o basalto, con suelos someros de arenisca o lateríticos, con frecuencia junto a vertientes y pequeños cursos de agua. Crece principalmente asociado a hierbas heliófilas como Andropogon bicornis L., Axonopus suffultus (J.C. Mikan ex Trin.) Parodi y especies de los géneros Eryngium y Tibouchina, entre otras. Ha sido hallada también en pastizales húmedos colonizados por especies leñosas como Baccharis dracunculifolia DC., e inclusive se la ha encontrado en el borde de la banquina sobre la ruta.

Según Pedron et al. (2012) esta especie es polinizada por lepidópteros esfíngidos del género Manduca Hübner, [1807].

Observaciones. Esta especie pertenece a la sección Macroceratitae Kraenzl. y es morfológicamente afín a $H$. gourlieana Gillies ex Lindl., de la cual se diferencia por los caracteres indicados en la clave. Véase Batista et al. (2006: 22) para una descripción más detallada y la lista sinonímica completa.
Material estudiado. ARGENTINA. Prov. Corrientes: Dpto. Ituzaingó, Establecimiento Santo Domingo, 19-XI-2014, Keller \& Franco 12451 (CTES). Prov. Misiones: Dpto. Candelaria, Ruta 105, a $15 \mathrm{~km}$ al W del Arroyo Mártires, 4-II-1976, Romanczuk et al. 755 (SI). Dpto. Iguazú, Arroyo Tupicuá, represa privada, 27-I-1983, Guaglianone et al. 1017 (SI). Dpto. San Ignacio, Paraje Teyú Cuaré, 16-VII-2015, Keller \& Ramírez 12832 (CTES).

2. Habenaria josephensis Barb. Rodr. Gen. Sp. Orchid. 2: 257. 1882. Tipo. Barbosa Rodrigues, Iconographie des Orchidées du Brésil 1: tab. 19. 1996 (lectotipo designado por Batista et al., Harvard Pap. Bot. 2011a: 38. (Fig. 1D-F).

Hierbas terrestres de $60-100 \mathrm{~cm}$ de altura. Tubérculos oblongo-elipsoides de ca. $5 \times 2 \mathrm{~cm}$. Hojas lanceoladas, de 1,8-13 × 0,5-2,3 cm, de ápice agudo, membranáceas. Inflorescencias laxas, pedúnculo de 1,6-3,5 cm de long., raquis de 16-22 $\mathrm{cm}$ de long.; brácteas lanceoladas de 1-2 × 0,3$0,6 \mathrm{~cm}$, de ápice agudo. Flores verdosas; sépalo dorsal ovado-orbicular, de 3-4 x 3-4 mm, ápice obtuso; sépalos laterales ovados, falcados, de 4-6 $\times$ 2-3 $\mathrm{mm}$, de ápice obtuso; pétalos 2-partidos, segmento posterior oblongo-lanceolado, falcado, de 3-4 $\times 1-2 \mathrm{~mm}$, de ápice obtuso, segmento anterior estrechamente oblongo, a veces reducido a un dentículo de 1-2 mm de long., de ápice agudo; labelo con segmentos laterales reducidos a dentículos de ca. $1 \mathrm{~mm}$ de long., de ápice obtuso, segmento medio estrechamente oblongo, de $6-8 \times 1 \mathrm{~mm}$, de ápice redondeado, espolón suberecto, de 0,9-1,2 cm de long. Gimnostemio de ca. $2 \mathrm{~mm}$ de long.; ovario y pedicelo de ca. $1,5 \mathrm{~cm}$ de long. Fruto cápsula 3 -costada, de 1,7-2,1 × 0,8-1,2 cm.

Iconografia. Vieira \& de Barros (2017: 720, fig. 6i); Hoehne (1940: tab. 41).

Distribución geográfica y hábitat. H. josephensis se encuentra citada con anterioridad como especie endémica para el Brasil (Batista et al., 2011; Vieira \& de Barros, 2017) en donde se la encuentra en selvas de galería y en la floresta estacional semidecídua (Vieira \& de Barros, 2017). En la Argentina (provincia de Misiones; Fig. 2) ha sido hallada solo en la selva marginal de un bañado abierto donde crece principalmente bajo la sombra de especies 


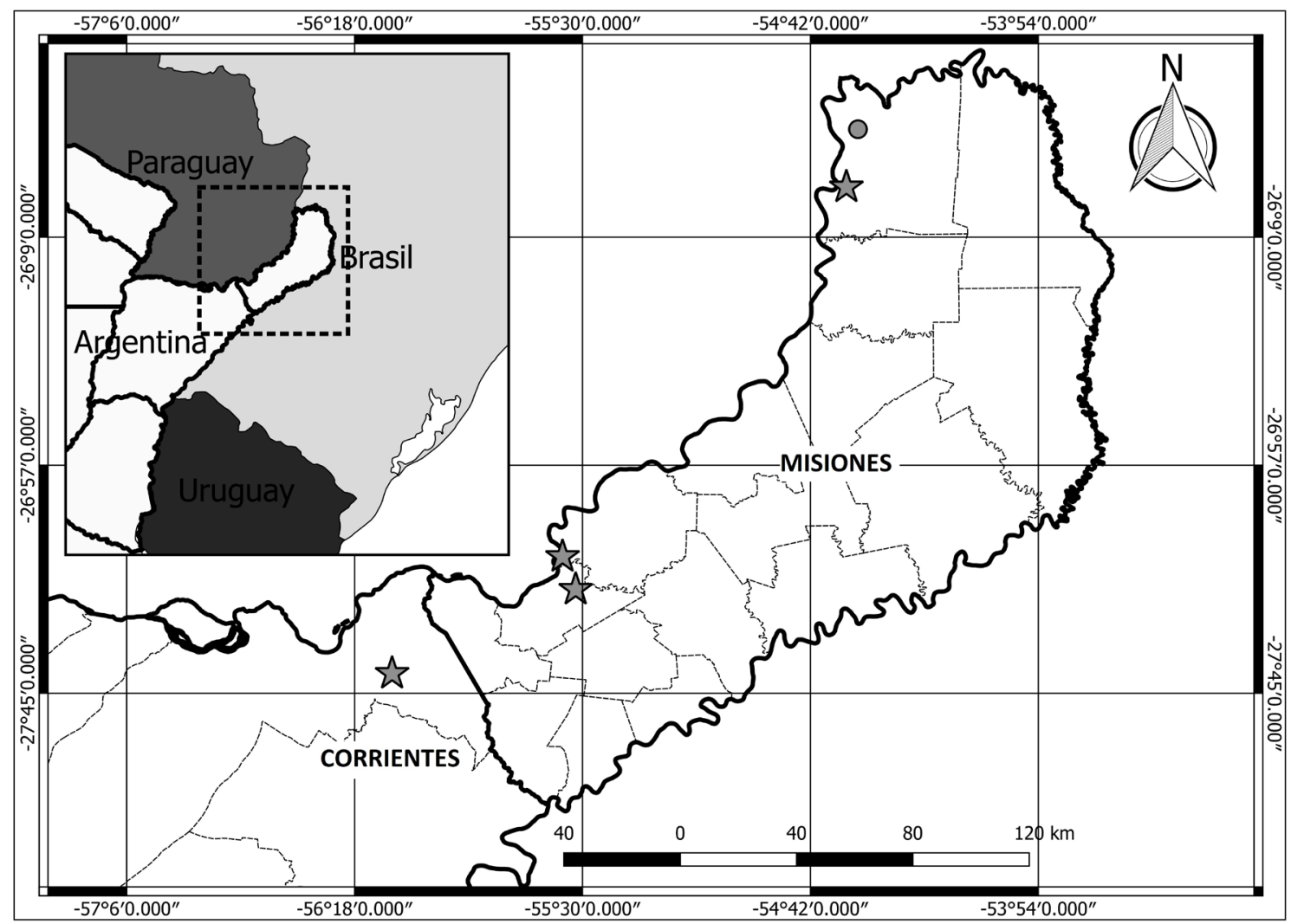

Fig. 2. Mapa de distribución de Habenaria johannensis (estrellas) y H. josephensis (círculo) en la Argentina.

pertenecientes a la familia Lauraceae y de Guadua trinii (Nees) Nees ex Rupr., así como también especies del estrato intermedio como Cordyline spectabilis Kunth \& C.D. Bouché. Con una población de unos veinte ejemplares, conforma parte del estrato basal del sotobosque, acompañado principalmente de helechos como Ctenitis submarginalis (Langsd. \& Fisch.) Ching además de hierbas esciófilas como Oplismenus hirtellus (L.) P. Beauv. El suelo sobre el cual arraiga es húmedo, pero no anegado, laterítico rojo tornándose gris en proximidades al sector pantanoso abierto del humedal.

Observaciones. Esta especie pertenece a la sección Micranthae Kraenzl. Véase Batista et al. (2011: 38) para la lista sinonímica completa.

Material estudiado. ARGENTINA. Prov. Misiones: Depto. Iguazú, Ruta Nacional 12, 9-V2015, Keller \& Radins 12823 (CTES).
Clave para diferenciar las especies de Habenaria en el área de estudio

1. Pétalos enteros. 2

1'. Pétalos 2-partidos. 4

2. Pétalos espatulados. Habenaria petalodes

2'. Pétalos triangular-lanceolados. 3

3. Labelo con el lóbulo medio más corto que los laterales; espolón sacciforme de 1-2(-3) $\mathrm{mm}$ de long. Habenaria guilleminii

3'. Labelo con el lóbulo medio más largo que los laterales; espolón claviforme de 10-12 mm de long. Habenaria hieromyni

4. Longitud total del segmento anterior del pétalo sobrepasando 1,8-2,6 veces la longitud del segmento posterior. 5

4'. Longitud total del segmento anterior del pétalo nunca sobrepasando 1,8 veces la longitud del segmento posterior. .. 6 
5. Flores verdosas; segmentos posteriores de los pétalos de 4-7 $\mathrm{mm}$ de ancho; lóbulo medio del rostelo prominente, proyectado más allá de las anteras; rostelo con el ápice ancho y dentado.

Habenaria johannensis

5'. Flores blanquecinas; segmentos posteriores de los pétalos de 2-2,5 $\mathrm{mm}$ de ancho; lóbulo medio del rostelo parcial o totalmente encerrado entre las anteras; rostelo con el ápice estrecho, agudo a obtuso.

Habenaria gourlieana

6. Longitud del segmento anterior del pétalo más corto que el posterior. 7

6'. Longitud del segmento anterior del pétalo de ca. igual longitud o apenas más largo que el posterior. 10

7. Labelo con los lóbulos laterales reducidos a dentículos de ca. $1 \mathrm{~mm}$ de long. Habenaria josephensis

7'. Labelo con los lóbulos laterales no reducidos, de 2,5-4 mm de long. .. 8

8. Inflorescencias laxas, paucifloras, de no más de 4 flores. Habenaria regnellii

8. -Inflorescencias densas, multifloras, de más de 4 flores. 9

9. Pétalos laterales y labelo blancos; sépalo dorsal de 7-8 mm de long.; sépalos laterales de 8-10 $\mathrm{mm}$ de long. Habenaria hassleriana

9'. Pétalos laterales y labelo verdosos; sépalo dorsal de 3-5 mm de long.; sépalos laterales de 3,5-5 $\mathrm{mm}$ de long. Habenaria parviflora

10. Pétalos laterales de 10-16 mm de long. ...... 11

10'. Pétalos laterales de 3,5-7 $\mathrm{mm}$ de long. ..... 12

11. Pétalo con el segmento posterior linear, de 1,2-1,5 $\mathrm{mm}$ de ancho, de aproximadamente el mismo ancho que el segmento anterior; inflorescencias densas multifloras de más de cinco flores.

Habenaria bractescens

11'. Pétalo con el segmento posterior lanceolado, de 2,5-3 $\mathrm{mm}$ de ancho, de aproximadamente el doble de ancho que el segmento anterior; inflorescencias laxas paucifloras de no más de cinco flores Habenaria trifida

12. Flores con el centro blanco y lóbulos de los pétalos y labelos verdes. 13

$12^{\prime}$. Flores con el centro blanco y lóbulos de los pétalos y labelos amarillos. 14

13. Pétalos con los segmentos posteriores sobrepasando al sépalo dorsal.

Habenaria araneiflora

13'. Pétalos con los segmentos posteriores nunca sobrepasando al sépalo dorsal.

Habenaria achalensis

14. Sépalos laterales de 3,5-4,5 de ancho; plantas de $80-150 \mathrm{~cm}$ de alto.

Habenaria ekmaniana

14'. Sépalos laterales de 2-3 $\mathrm{mm}$ de ancho; plantas de $10-50 \mathrm{~cm}$ de alto. 15

15. Espolón claviforme; hojas linear-lanceoladas de 0,6-1 $\mathrm{cm}$ de ancho.

Habenaria pentadactyla

15'. Espolón filiforme; hojas lanceoladas de 1,5$2 \mathrm{~cm}$ de ancho. 16

16. Plantas acuáticas, con estolones.

Habenaria repens

16'. Plantas terrestres, sin estolones.

Habenaria aranifera

Para las especies Habenaria achalensis (Fig. 3A), H. araneiflora (Fig. 3B), H. bractescens (Fig. 3C), H. ekmaniana (Fig. 3D), H. gourlieana (Fig. 3E), H. parviflora (Fig. 3F), H. regnellii (Fig. 3G) y H. trifida (Fig. 3H), se ilustran algunos de los caracteres morfológicos para ayudar a su identificación.

\section{Agradecimientos}

A Maria Salete Marchioretto (PACA) por el envío de material fotográfico de ejemplares. A Diego Gutiérrez y a un revisor anónimo por sus revisiones y sugerencias para enriquecer el manuscrito. 
C. A. Zanotti et al. - Dos nuevos registros de Habenaria para Argentina

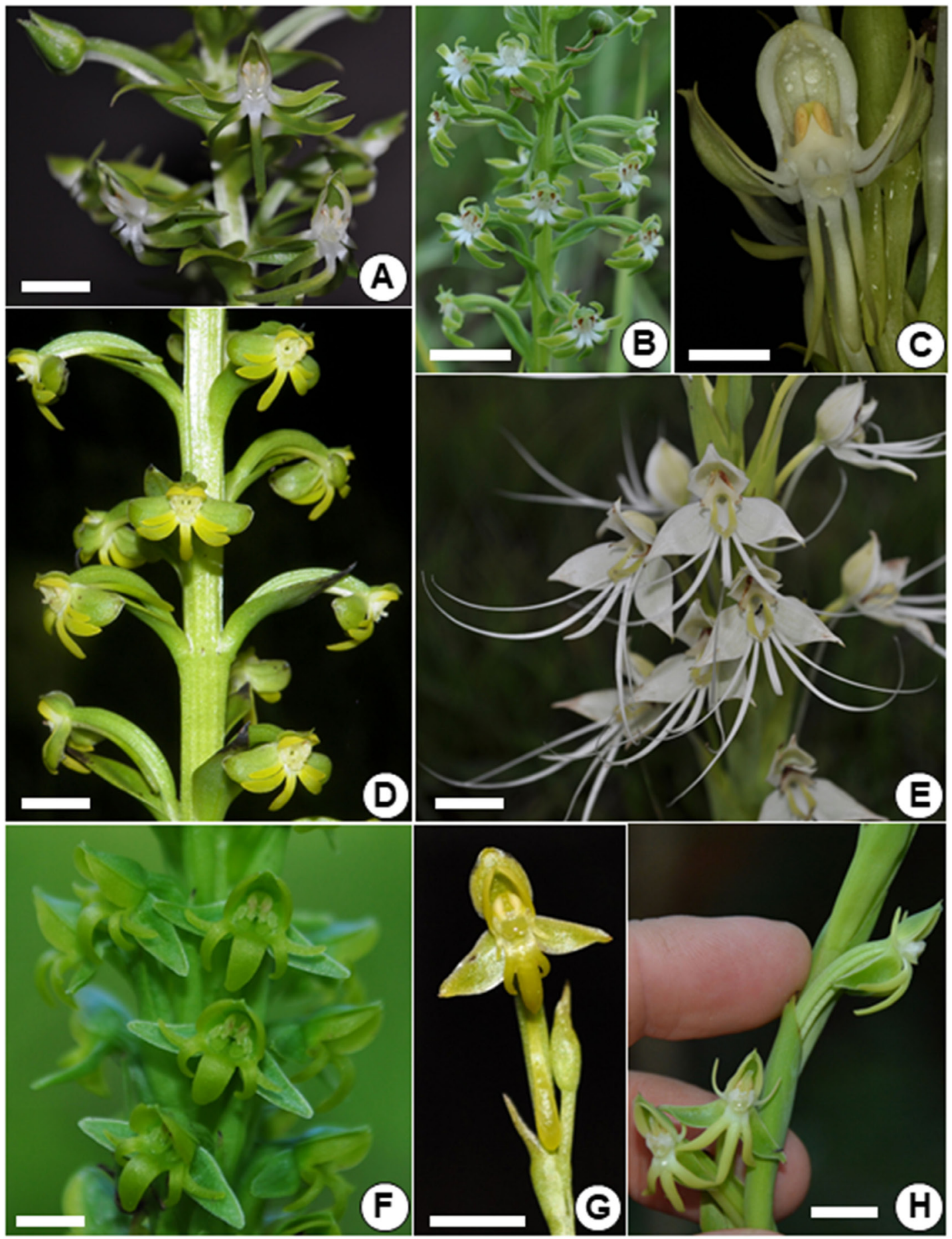

Fig. 3. A-H: Diversidad floral de Habenaria de Argentina. A: Habenaria achalensis. B: $H$. araneiflora. C: $H$. bractescens. D: H. ekmaniana. E: H. gourlieana. F: H. parviflora. G: H. regnellii. H: H. trifida. Escalas $=$ A: 6 mm; B: $21 \mathrm{~mm}$; C: 10 mm; D: 7 mm; E: 20 mm; F: 1,5 mm; G: 8 mm; H: 5 mm. Fotos: A-B, D-H: J. A. Radins, C: C. A. Zanotti. 


\section{Biblografía}

BATEMAN, R. M., P. M. HOLLINGSWORTH, J. PRESTON, L. YI-BO, A. M. PRIDGEON \& M. W. CHASE. 2003. Molecular phylogenetics and evolution of Orchidinae and selected Habenariinae (Orchidaceae). Bot. J. Linn. Soc. 142: 1-40.

BATISTA, J. A. N., L. B. BIANCHETTI \& Z. J. G. MIRANDA. 2006. A revision of Habenaria section Macroceratitae (Orchidaceae) in Brazil. Brittonia 58: $10-41$.

BATISTA, J. A. N., L. B. BIANCHETTI, R. GONZÁlEZ-TAMAYO, X. M. C. FIGUEROA \& P. J. CRIBB. 2011a. A synopsis of new world Habenaria (Orchidaceae) I. Harvard Pap. Bot. 16: $1-47$.

BATISTA, J. A. N., L. B. BIANCHETTI, R. GONZÁlEZ-TAMAYO, X. M. C. FIGUEROA \& P. J. CRIBB. 2011b. A synopsis of new world Habenaria (Orchidaceae) I. Harvard Pap. Bot. 16: 233-273.

BAtistA, J. A. N., K. S. BORGES, M. W., M. W. FARIA, K. PROITE, A. J. RAMALHO, G. A. SALAZAR \& C. VAN DEN BERG. 2013. Molecular phylogenetics of the species-rich genus Habenaria (Orchidaceae) in the New World based on nuclear and plastid DNA sequences. Molec. Phylogen. Evol. 67: 95-109.

COGNIAUX, A. 1893. Orchidaceae. Habenaria (Orchidaceae). In: Martius, C. F. P. von, A. W. Eichler \& I. Urban (eds.). Flora Brasiliensis. 3(4): 18-102. F. Fleischer, Munchen, Wien, Leipzig

DRESSLER, R. L. 1993. Phylogeny and classification of the orchid family. Dioscorides Press, Portland.

GOVAERTS, R., P. BERNET, K. KRATOCHVIL, G. GERLACH, G. CARR, P. ALRICH, A. M. PRIDGEON, J. PFAHL, M. A. CAMPACCI, D. HOLLAND BAPTISTA, H. TIGGES, J. SHAW, P. J. CRIBB, A. GEORGE, K. KREUZ \& J. WOOD. 2018. World checklist of Orchidaceae. Kew: Royal Botanic Gardens [on line]. Disponible en: http:// wcsp.science.kew.org/ [acceso 25 Marzo 2018].
HOEHNE, F. C. 1940. Habenaria. In: Flora Brasilica. 12: 52-254. Secretaria da Agricultura, Industria e Comércio da São Paulo, San Pablo.

KRÄNZLIN, F. 1892. Beitrage zu einer monographie der gattung Habenaria Willd. Bot. Jahrb. Syst. 16: 52-223.

KRÄNZLIN, F. 1901. Orchidaceum genera et species. Vol. 1 Habenaria. Mayer \& Müller, Berlín.

KURZWEIL, H. \& A. WEBER. 1992. Floral morphology of southern African Orchideae. II. Habenariinae. Nordic J. Bot. 12: 39-61.

PEDRON, M., C. R. BUZATTO, R. B. SINGER, J. A. N. BATISTA \& A. MOSER. 2012. Pollination biology of four sympatric species of Habenaria (Orchidaceae: Orchidinae) from southern Brazil. Bot. J. Linn. Soc. 170: 141-156.

PEDRON, M., C. A. BUZATTO, A. J. RAMALHO, B. M. CARVALHO, J. A. RADINS, R. B. SINGER \& J. A. N. BATISTA. 2014. Molecular phylogenetics and taxonomic revision of Habenaria section Pentadactylae (Orchidaceae, Orchidinae). Bot. J. Linn. Soc. 175: 47-73.

PRIDGEON, A. M., P. J. CRIBB, M. W. CHASE, F. N. RASMUSSEN. 2001. Genera orchidacearum, vol. 2, Orchidoideae, part 1. Oxford University Press, New York.

SCHININI, A., J. WAECHTER, P. IZAGUIRRE \& C. LEHNEBACH. 2008. Orchidaceae. En: Zuloaga, F. O, O. Morrone \& M. J. Belgrano (eds.), Catálogo de las Plantas vasculares del Cono Sur I. Monogr. Syst. Bot. Missouri Bot. Gard. 107: 472-609.

SINGER, R. B. \& A. A. COCUCCI. 1997. Eye attached hemipollinaria in the hawkmoth and settling moth pollination of Habenaria (Orchidaceae): a study on functional morphology in five species from subtropical South America. Bot. Acta 110: 328-337.

VIEIRA, T. L. \& F. DE BARROS. 2017. Orchidaceae na Serra do Ouro Branco, Minas Gerais, Brasil. Rodriguésia 68: 691-747.

Recibido el 23 de enero de 2018, aceptado el 19 de marzo de 2018. Editor: Diego Gutiérrez. 